\title{
A Novel Adaptive Fuzzy Autopilot Design for ASV Tracking Control
}

\author{
M.H. Wang, Y.Q. Yu, W. Lin \\ Faculty of Computer Science \\ Guangdong University of Technology \\ Guangzhou, China
}

\begin{abstract}
A novel robust adaptive fuzzy control algorithm is presented for autonomous surface vehicle (ASV) autopilot. This paper studies an approach for fuzzy rule base optimization. The optimization solution especially solves the non-compatible problem in the generation process of fuzzy control rules. For the design study, the optimization model has been carried out in the simulink environment. It is shown that the proposed algorithm guarantees that the tracking control system is asymptotically stable and its tracking error can approach to zero.Simulation results also show the effectiveness of the algorithm in the presence of disturbances, and high performance of the proposed controller.
\end{abstract}

Keywords- autonomous surface vehicle; fuzzy control; tracking control; simulation

\section{INTRODUCTION}

To design an autopilot for ASV is always a challenging problem. An ASV dynamics is influenced by unpredictable environmental disturbances such as waves, winds, currents, change of depth under keel, etc. as well as ASV sailing conditions such as speed, loading condition, trim, etc. Automatic steering of ASVs has been a goal of seafarers for many years. Imperatives such as reduced manning and increasing fuel costs has led to innovative designs from the classic PID controller to adaptive and robust control and latterly to intelligent control which is the focus of this paper.

With the development of control theory and method, the control of autopilot is gradually improved. Traditional control techniques have encountered difficulties. PID autopilot lacks the adaptability to the change of the working state and environment of the ASV, so it is hard to achieve optimal steering since the parameters of autopilot have to be set manually. adaptive autopilot[1] has certain adaptability to the change of the working state and environment of the ASV, and can automatically adjust the control parameters. But PID autopilot and self-adaptive autopilot are based on the accurate mathematical model, while the actual ASV steering process changes with the working situations (load, draft depth, navigational speed) and navigational environments (such as navigational route, wind, wave and flow and so on). So the traditional control cannot avoid the problems, such as difficult adjustment of controller parameters, and the robustness. To address the above problems, the intelligent control technologies such as fuzzy control[2-4], neural network[5-9] and genetic algorithm[10] were gradually used in the course control, since the intelligent control technology requires no mathematical model and the system has good robustness.

\section{FUZZY CONTROL SYSTEM FOR ASV AUTOPILOT}

The use of fuzzy set theory as a method for replicating the non-linear behavior of an experienced helmsman is perhaps the most appropriate application of this technique. Fuzzy rules of the type: heading error is positive small AND heading error rate is positive big THEN rudder angle is positive medium, typify the actions of an experienced helmsman. The schematic of a fuzzy logic controller is shown in Fig. 1 where the conventional controller block is replaced by a composite block comprising four components:

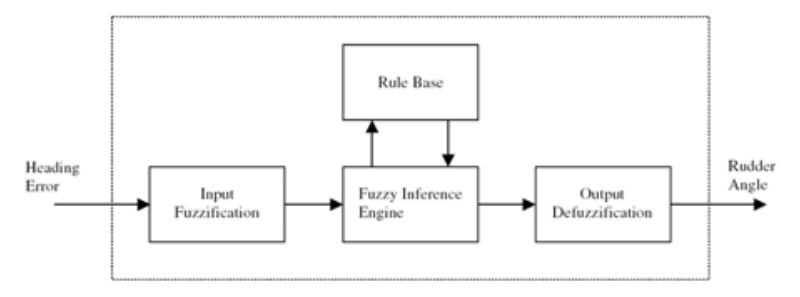

FIGURE I. FUZZY CONTROL FOR ASV AUTOPILOT

A fuzzy course controller is designed, where course deviation $e=\psi_{r}-\psi$ and heading rate $r=\dot{\psi}$ are two input quantities of fuzzy controller, and the rudder angle for the output command of the controller is $\delta_{\psi}$.

In practical applications, the control goals and system constraints are all of fuzzy characters, in order to unify them, fuzzy membership function is used to express their characters. These operators can be used to translate a linguistic description of control goals into a decision function. In this way, various forms of aggregation can be chosen giving greater flexibility for expressing the control goals. The universe of discourse (range) of the inputs and outputs are mapped into several fuzzy sets of desired shapes. The membership functions for the inputs are shown in Fig 2,3 and outputs are shown in Fig 4. 


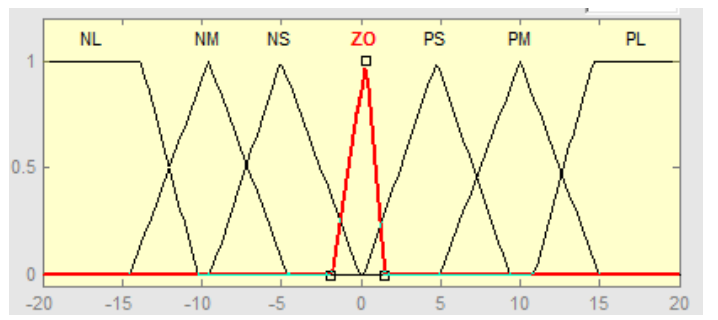

FIGURE II. MEMBERSHIP FUNCTIONS FOR YAW ERROR (E)

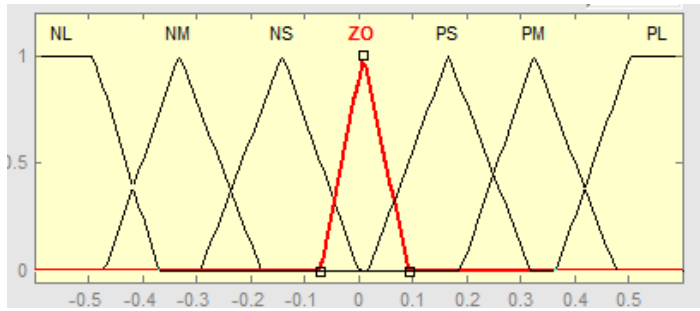

FIGURE III. MEMBERSHIP FUNCTIONS FOR YAW RATE $(\Omega)$

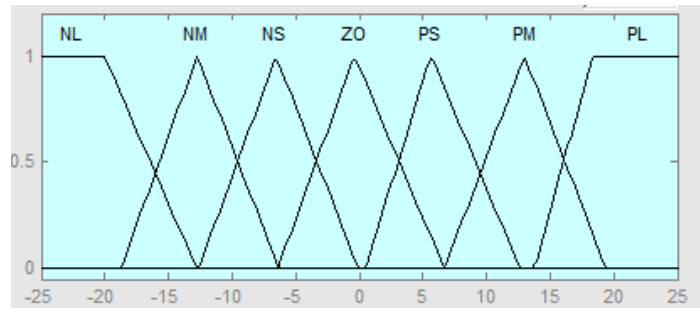

\section{FIGURE IV. MEMBERSHIP FUNCTIONS FOR RUDDER ANGLE $(\Delta \mathrm{C})$}

A fuzzy system is characterized by a set of linguistic statements based on expert knowledge. The expert knowledge is usually as "if-then" rules, which are easily implemented by fuzzy conditional statements in fuzzy logic. Fuzzy control rules have the form of fuzzy conditional statements that relate the state variables in the antecedent and process control variables in the consequence. Rules that were developed in the work are given in Table1.

\begin{tabular}{cccccccc}
\multicolumn{7}{c}{ TABLE I. RULES FOR ASV TRACKING CONTROL } \\
\hline \multirow{2}{*}{$\begin{array}{c}\text { Yaw } \\
\text { rate }\end{array}$} & NL & NM & NS & ZO & PS & PM & PL \\
\cline { 2 - 7 } NL & NL & NL & NL & NL & NL & NM & NS \\
NM & NL & NL & NL & NM & NM & NS & ZO \\
NS & NL & NL & NM & NM & NS & ZO & PL \\
ZO & NM & NM & NM & NS & ZO & PL & PL \\
PS & NM & NM & NS & ZO & PL & PL & PL \\
PM & NM & NS & ZO & PL & PL & PL & PL \\
PL & NS & ZO & PL & PL & PL & PL & PL \\
\hline
\end{tabular}

III. OPTIMIZATION OF FUZZY CONTROL RULE

The paper proposed a new method to solve the noncompatible problem in the generation process of fuzzy control rules, which is a common problem in practice.

Firstly, we analyze the compatibility problem of fuzzy rule.
Fuzzy set $\underset{\sim}{A}$ and $\underset{\sim}{B}$ are of the universe of discourse $\mathbf{X}$

$$
\begin{aligned}
& \underset{\sim}{A}=\left(\mu_{A}\left(x_{1}\right), \mu_{A}\left(x_{2}\right), \cdots, \mu_{A}\left(x_{m}\right)\right) \\
& B=\left(\mu_{B}\left(x_{1}\right), \mu_{B}\left(x_{2}\right), \cdots, \mu_{B}\left(x_{m}\right)\right)
\end{aligned}
$$

The lattice degree of nearness of $\underset{\sim}{A}$ and $B_{\sim}$ as following:

$$
\sigma(\underset{\sim}{A}, \underset{\sim}{B})=\frac{1}{2}(\underset{\sim}{A} \circ \underset{\sim}{B}+(1-\underset{\sim}{A} \otimes \underset{\sim}{B}))
$$

So the simple lattice degree of nearness can be obtained as following:

$$
\sigma(\underset{\sim}{A} \underset{\sim}{B})=\underset{\sim}{A} o \underset{\sim}{B}=\underset{l=1}{\vee}\left[{\underset{\sim}{\sim}}_{A}\left(x_{l}\right) \wedge \underset{\sim}{\mu_{B}}\left(x_{l}\right)\right]
$$

When the lattice degree of nearness $\sigma(A, B)=1$, $A_{\text {and }} B$ have strong compatibility. When the lattice degree of nearness $\sigma(\underset{\sim}{A}, B)=0, \quad A_{\sim}$ and $B$ are incompatible. When the lattice degree of nearness $\sigma \underset{\sim}{A} \underset{\sim}{B}) \in(0,1)$, $A_{\sim}$ and $B_{\sim}$ have strong compatibility.

So, the compatibility coefficient is adopted as the lattice degree of nearness. Fuzzy if-then rules for a $c$-class pattern classification problem with $n$ attributes can be written as

Rule $R_{k}$ : if $A_{i}^{k}$ and $B_{j}^{k}$ then $C_{i j}^{k}$

Rule $R_{k^{\prime}}$ : if $A_{\sim}^{k^{\prime}}$ and $B_{j}^{k^{\prime}}$ then $C_{i j}^{k^{\prime}}$

where $A_{i}, B_{j}$ is an antecedent linguistic value such as small and large ( $i \quad 1,2, \ldots, n), C_{i j}$ is a consequent class (i.e., one of the given $c$-classes), $k \quad\left(1,2, \ldots, \mathrm{n}^{2}\right)$, and $\mathrm{n}$ is the number of fuzzy if -then rules.

$$
\begin{gathered}
\operatorname{c}_{\sim}^{k} \times B_{j}^{k} \rightarrow C_{\sim}^{k} \\
P_{\sim}^{k}\left(x_{l}\right)={\underset{\sim}{\sim}}_{\sim}^{k} \times B_{j}^{k}=A_{i}^{k}\left(x_{l}\right) \wedge B_{j}^{k}\left(x_{l}\right)
\end{gathered}
$$

The compatibility coefficient of the antecedents between rule $k$ and rule $k$ is obtained as following:

$$
\begin{aligned}
& \sigma\left(P_{\sim}^{k}, P_{\sim}^{k^{\prime}}\right)=\underset{l=1}{\vee}\left[P_{\sim}^{k}\left(x_{l}\right) \wedge P_{\sim}^{k^{\prime}}\left(x_{l}\right)\right] \\
& =\left\{\underset{l=1}{\stackrel{m}{\vee}}\left[\sim_{\sim}^{k}\left(x_{l}\right) \wedge B_{j}^{k}\left(x_{l}\right)\right]\right\} \wedge\left\{\underset{l=1}{\stackrel{m}{\vee}}\left[A_{l}^{k^{\prime}}\left(x_{l}\right) \wedge B_{j}^{k^{\prime}}\left(x_{l}\right)\right]\right\}
\end{aligned}
$$

The compatibility coefficient of the consequents between rule $k$ and rule $k^{\prime}$ is also obtained:

$$
\sigma\left(C_{\sim}^{k}, C_{\sim}^{k^{\prime}}\right)=\underset{l=1}{\stackrel{m}{\vee}}\left[C_{i j}^{k}\left(y_{l}\right) \wedge C_{\sim}^{k_{i j}^{\prime}}\left(y_{l}\right)\right]
$$


According to the compatibility principle of fuzzy rule table, we have

$$
\delta_{k k^{\prime}}=\left|\sigma\left(\underset{\sim}{P^{k}, P_{\sim}^{k^{\prime}}}\right)-\sigma\left(\underset{\sim}{C_{\sim}^{k}}, C_{\sim}^{k^{\prime}}\right)\right|
$$

The maximal compatibility coefficient is a compatibility class that is not properly contained between two compatibility class. One of two rule must be redundant or rough to be needed to modify.

In order to find which rule is redundant or rough between the two non-compatible rules. Considered between rule $\mathrm{k}$ with any other rule, the compatibility coefficient of rule $k$ is calculated:

$$
\delta_{k}=\sum_{k^{\prime}=1}^{n^{2}} \delta_{k k^{\prime}}
$$

The maximal compatibility coefficient means the rule has the least compatibility with any other rule. So we modify consequent class of the rule in order to make ITAE least.

Secondly, we establish the fuzzy matter-element for compatibility problem.

$\tilde{R}=($ fuzzy rule, compatibility, compatibility coefficient)

$$
\tilde{R}_{k}=\left[\begin{array}{ccc}
k, & c_{1}, & v_{1} \\
c_{2}, & v_{2} \\
\vdots & \vdots \\
c_{k}, & v_{k} \\
\vdots & \vdots \\
c_{n}, & v_{n}
\end{array}\right]
$$

where $\mathrm{k}$ is fuzzy rule $(\mathrm{k}=1,2, \ldots, \mathrm{n}), \mathrm{c}$ is the property of compatibility. $v_{k}$, is the compatibility coefficient

$$
\sigma_{k k^{\prime}}\left(k^{\prime}=1,2, \ldots n\right) \text {. }
$$

Secondly, we establish the dependent function $K(\tilde{R})$.

$$
K(\tilde{R})=\frac{\sigma_{k^{\prime}}-\sigma_{k}}{\sigma_{k k^{\prime}}}
$$

In the positive region

$$
\tilde{A_{+}}(\tilde{R})=\{\tilde{R} \mid \tilde{R} \in \tilde{W}, K(\tilde{R}) \geq 0\},
$$

It means rule $k$ has better compatibility than rule $k^{\prime}$, so rule $k$ needn't to be modified.

\section{Simulation Result}

Simulation environment is used under Matlab environment to realize an integrated environment with modeling and simulation from the designed intelligent controller to the whole control system. Firstly visualization modeling, build the model of the control system, before simulation and commissioning. In the simulation system can realize the file interchange and data exchange among various working environments.
Navigation of the ASV in wind and wave requires the controller to have the resistance against interference. Because of frequently happening speed change, there will be the model parameter perturbation, so the controller is required to have the robustness and resistance against interference. What follows will be the simulation of the controller with parameter perturbation and wind and wave interference. The yaw response of the ANFIS controller was shown in Figure 5.

Figure 5 shows that the yaw angle errors between the desired and obtained yaw responses. It can be observed that the fuzzy controller designed obtains a perfect performance for tracking under the worst case conditions which exist random disturbance.

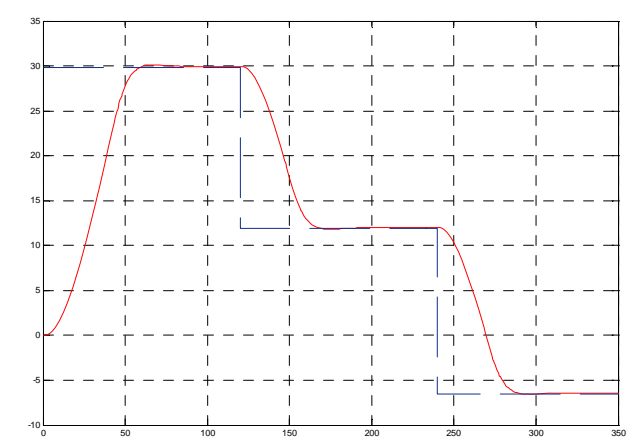

FIGURE V. THE YAW RESPONSE FOR ASV TRACKING

\section{CONCLUSION}

In this paper, the tracking control problem has been considered for a class of uncertain nonlinear systems with the unknown system function and unknown gain function, and fuzzy logic systems have been used to approximate unknown system function and a robust adaptive fuzzy tracking control algorithm. In order to improve the performance of autopilot, this paper studies fuzzy rule base optimization. Moreover, a new adaptive control designing scheme for ASV tracking was proposed. The optimized result obtained is more suitable to the actual situation. Simulation shows that the above algorithm is feasible.

\section{REFERENCES}

[1] Guan Wei, Zhang Xian-ku,Concise nonlinear adaptive robust control of ship steering systems, IEEE International Conference on Automation and Logistics,pp.343 - 346, 2009.

[2] Fauadi, H.F. ; Nordin, M.H. ;Frontal obstacle avoidance of an autonomous subsurface vehicle (ASV) using fuzzy logic method, International Conference on Intelligent and Advanced Systems, pp. 125$128,2007$.

[3] Wang Minghui ; Yu Yongquan ;Optimization of fuzzy control system based on extension method for ship course-changing/keeping ,IEEE World Congress on Computational Intelligence,pp. 434-438,2008

[4] Perera, L.P. ; Carvalho, J.P. ;Solutions to the Failures and Limitations of Mamdani Fuzzy Inference in Ship Navigation, IEEE Transactions on Vehicular Technology , 63 (4),pp. 1539 - 1554,2014

[5] Xingxing Huo ; Jiangqiang $\mathrm{Hu}$; Zeyu Li , Technologies BP neural network based PID control for ship steering, World Congress on Information and Communication, pp. 1042 - 1046,2012

[6] Yanxiang Wu ; Minjie Xue ; Pengjie Wu,Study on fuzzy neural network-based ship autopilot, International Conference on Natural Computation, vol.3 pp. $1393-1396,2010$ 
[7] Zeyu Li ; Jiangqiang Hu ; Xingxing Huo,PID control based on RBF neural network for ship steering, World Congress on Information and Communication Technologies, pp.1076 - 1080,2012

[8] Jin Zhao ; Huajun Zhang,The application of fuzzy neural network in ship course control system, International Conference on Fuzzy Systems and Knowledge Discovery,vol.2, pp.752 - 756,2010

[9] Yong Cai ; Yunfei Lv ; Hui Luo,The application of fuzzy neural network in ship course control system, International Workshop on Advanced Computational Intelligence,pp. 338 - 342,2011

[10] $\mathrm{Fu}, \mathrm{He}-\mathrm{he} ; \mathrm{Li}$, Zhi-jun ; Modeling and controlling of engineering ship based on genetic algorithm, Modelling, Proceedings of International Conference on Identification \& Control, PP.394 - 398,2012 